\title{
Fission of Heavy Elements Induced by Medium High Energy Protons
}

\author{
Alexis C. Pappas \\ Department of Chemistry, University of Oslo, Blindern, Norway \\ (Z. Naturforschg. 21 a, 995-1001 [1966] ; received 21 March 1966)
}

Dedicated to Professor Dr. W. Gentner on the occasion of his 60th birthday

\begin{abstract}
Fissionability, mass distribution, charge dispersion and distribution, and neutron emission for fission induced in heavy targets (uranium) by protons with a few hundred $\mathrm{MeV}$ kinetic energy are presented and phenomenologically discussed. The results are interpreted in order to arrive at the reaction mechanisms in medium high energy induced fission.
\end{abstract}

In this paper is presented a discussion of fission induced in heavy elements (uranium) by protons with kinetic energies below about $400 \mathrm{MeV}$ with the main emphasis on fission with protons of a few hundred $\mathrm{MeV}$ energy *. Thus, reactions where meson interference becomes of any importance are not considered. Even if fission is the main reaction in heavy nuclei this can not be discussed without consideration of the cascade-evaporation process generally considered to take place at high energy interactions with complex nuclei.

As described by the Serber ${ }^{1}$ model these reactions start with a fast nucleonic cascade (time scale $\sim 10^{-22} \mathrm{sec}$ ) in the target nuclei knocking out neutrons and protons and leading to a varity of product nuclei - each with broad distribution of excitation energies - cascade residuals.

The subsequent deexcitation of the cascade residuals takes place by successive evaporations of nucleons (time scale $\gg 10^{-20} \mathrm{sec}$ ), each with a few $\mathrm{MeV}$ of kinetic energy (in heavy targets mainly neutrons) and leaving behind a complex mixture of reaction products - spallation products. Both steps of this model have been treated by the Monte Carlo $\operatorname{method}^{2-5}$.

The results of the calculations are in good agreement with the experimental data for the light particles knocked out and emitted in the two steps at energies of interest for the present study.

* For a comprehensive review of nuclear reactions including fission at high bombardment energies, see E. K. Hyde, The Nuclear Properties of the Heavy Elements III, Fission Phenomena, Chapter II, Prentice Hall Inc., Englewood Clifts, N. J. 1964.

1 R. Serber, Phys. Rev. 72, 1114 [1947].

2 N. Metropolis, R. Bivins, M. Strom, A. Turkevich, J. M. Miller, and G. Friedlander, Phys. Rev. 110, 185 [1958].

3 N. Metropolis, R. Bivins, M. Strom, J. M. Miller, G. Friedlander, and A.Turkevich, Phys. Rev. 110, 204 [1958].
The spectrum and yields of the spallation products from heavy targets as estimated according to this model are, however, upper limits, because in targets such as trans lead elements an additional difficulty enters the spallation picture. This is caused by a severe competition from fission during each stage of the evaporation chain, which lowers the yields of the spallation products. Such interference has also been treated by Monte Carlo methods ${ }^{6,7}$.

Thus high energy induced reactions in heavy target nuclei will result in a very large variety of

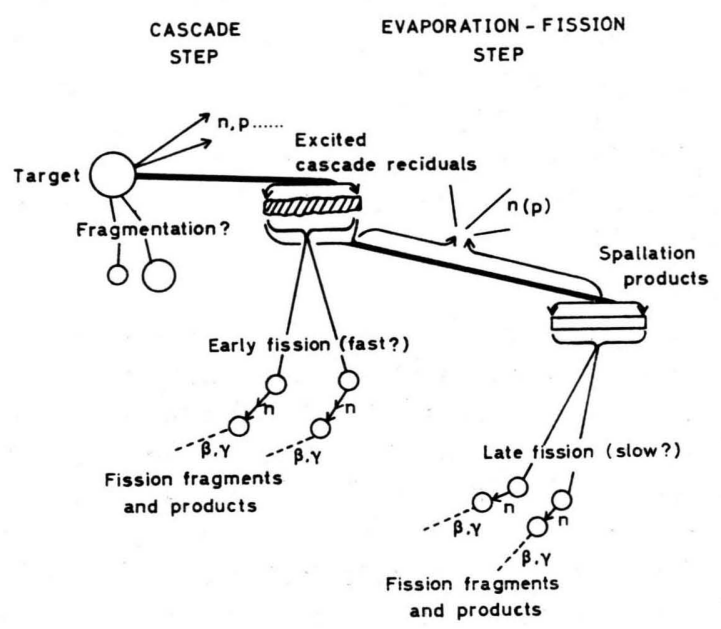

Fig. 1. Schematic representation of mechanisms of high energy induced reactions in heavy nuclei.

4 I. Dostrovsky, P. Rabinowitz, and R. Bivins, Phys. Rev. 111, 1659 [1958].

5 I. Dostrovsky, Z. Fraenkel, and G. Friedlander, Phys. Rev. 116, 683 [1959].

${ }^{6}$ I. Dostrovsky, Z. Fraenkel, and P. Rabinowitz, Proc. 2nd Intern. Conf. Peaceful Uses of Atomic Energy 15, 301, United Nations, Geneva 1958.

7 M. Lindner and A. Turkevich, Phys. Rev. 119, 1632 [1960]. 
heavy primary reaction products as result of the nucleonic cascade and the evaporation-fission competition. Furthermore, the primary fission fragments will de-excite by neutron evaporation, and the number of evaporated neutrons may in extreme cases be rather large (Fig. 1).

Even if spallation and fission each results in a complex mixture of products covering broad regions in the "Table of Nuclides", the "region of interest" for the different processes can be distinguished to some extent, depending on the energy of the bombarding particle. For uranium and bombarding energies below about $4.00 \mathrm{MeV}$ the fission region is clearly defined to the mass number region $60-160$. Results to be presented in this paper are mainly obtained through radiochemical analysis, but results from ionization chamber measurements and loaded emulsion studies are included to some extent as a supplement to the radiochemical studies.

\section{Fissionability}

According to the Monte Carlo calculations ${ }^{2,3}$ the total inelastic cross section $\sigma_{\mathrm{T}}$ should be nearly independent of energy above $100 \mathrm{MeV}$, in agreement with experimental data ${ }^{8}$. Due, however, to nuclear transparency ${ }^{2}$ this cross-section is expected to be slightly below the geometrical one, $\sigma_{\text {geom. }}$, for heavy elements at these energies, but to a first approximation and within the experimental errors not far from $\sigma_{\text {geom. }}$.

The fission cross sections at different energies and targets as measured by chemical and by physical methods scatter somewhat, but with a few exceptions these are in agreement within $20 \%$ or less ${ }^{9}$. As pointed out by Perfilov ${ }^{10}$ and by Pappas ${ }^{11}$, using average values and total inelastic cross sections, the fissionability for elements tantalum and heavier, defined as $\sigma_{\mathrm{f}} / \sigma_{\mathrm{T}}$, increases with proton energy ultimately level off or for trans lead elements reaches

8 G. P. Milburn, W. Birnbaum, W. E. Crandall, and L. Schechter, Phys. Rev. 95, 1268 [1954].

9 H. G. de Carvalho, G. Cortini, M. Muchnik, G. Potenza, R. Rinzivillo, and W. O. Lock, Nuovo Cim. 27, 468 [1963].

10 N. A. Perfilov, Soviet Phys.-JETP 14, 623 [1962].

11 A. C. Pappas, unpublished, presented at the Conference on High Energy Reactions in Complex Nuclei, Leysin 1963.

12 R. Wolfgang, E. W. Baker, A. A. Caretto, J. B. Cumming, G. Friedlander, and J. Hudis, Phys. Rev. 103, 394 [1956].

13 A. KJelberg and A. C. Pappas, Nucl. Phys. 1, 322 [1956]. a flat broad maximum whereafter it decreases steadily. The decrease may primarily be ascribed to increasing interference of other reactions caused by meson production. This becomes of particular importance in heavy elements where many of the pions produced have a chance to be reabsorbed within the mother nucleus ${ }^{12}$.

Thus the maximum contribution from fission is found at bombarding energies 100 to $300 \mathrm{MeV}$ for thorium and uranium, but at considerably higher energies, $400-800 \mathrm{MeV}$, for bismuth.

Elements thorium and heavier belong to the highly fissionable ones where fission predominates over spallation and other reactions $\left(\sigma_{\mathrm{f}} / \sigma_{\mathrm{T}}=0.5-1\right)$. These nuclei are all characterized by a low fission threshold, in the vicinity of neutron binding energies $(\sim 5 \mathrm{MeV})$. In the lead region fission is less probable $\left(\sigma_{\mathrm{f}} / \sigma_{\mathrm{T}}=0.05-0.1\right)$ and characterized by a high fission threshold, higher than neutron binding energies. For still lighter elements the fission thresholds are considerably higher than neutron binding energies and amount to tens of $\mathrm{MeV}$, and fission should become very unlikely compared to spallation for these elements.

The dependence of $\sigma_{\mathrm{f}} / \sigma_{\mathrm{T}}$ on nuclear size does not seem to be as strong in high energy induced fission as in fission induced by low energy (few $\mathrm{MeV}$ ) bombardments. As, however, in the latter case the former can be represented by a $Z^{2} / A$ dependence ${ }^{10,11}$ and the maxima in the $\sigma_{\mathrm{f}} / \sigma_{\mathrm{T}}$ versus energy curves are, according to Perfilov ${ }^{10}$, given by

$$
\left(\sigma_{\mathrm{f}} / \sigma_{\mathrm{T}}\right)_{\max } \propto \exp \left[0.682\left(Z^{2} / A-36.25\right)\right],
$$

with $\sigma_{\mathrm{T}}=\pi\left(1.26 \mathrm{~A}^{1 / 3}-0.41\right)^{2} \cdot 10^{-2} \mathrm{~b}$.

The maximum fissionability of uranium occurs in the energy region $100-300 \mathrm{MeV}$ and is about $80 \%$. Thus the studies by the author and his collaborators on fission of uranium with $170 \mathrm{MeV}$ protons ${ }^{13-19}$ fall in the middle of the maximum fissionability of uranium. The latest fission cross section measurements give $(1.4 \pm 0.1) \mathrm{b}$ at $170 \mathrm{MeV}$ measured

14 P. Aagand, G. Andersson, J. O. Burgman, and A. C. Pappas, J. Inorg. Nucl. Chem. 5, 105 [1957].

15 J. Alstad and A. C. PAppas, J. Inorg. Nucl. Chem. 15, 222 [1960].

16 A. C. Pappas and J. Alstad, J. Inorg. Nucl. Chem. 17, 195 [1961].

17 G. Rudstam and A. C. Pappas, Nucl. Phys. 22, 468 [1961].

18 E. Hagebø, A. C. Pappas, and P. Aagaard, J. Inorg. Nucl. Chem. 26, 1639 [1964]

19 A. C. Pappas and E. Hagebø, J. Inorg. Nucl. Chem, in press 1966. 
radiochemically ${ }^{19}, 1.37 \mathrm{~b}$ at $150 \mathrm{MeV}$ as determined by ionization chamber ${ }^{20}$, and $(1.46 \pm 0.06) \mathrm{b}$ at $280 \mathrm{MeV}$ based on fragment track techniques ${ }^{21}$.

The fissionability for a given nucleus at a given excitation energy will increase when its angular momentum is increased ${ }^{22}$. Thus the fissionability in high energy proton bombardments should be expected to increase in those cases where large angular momenta are involved, but no separation of results from low and high angular momentum proton interactions have yet been made. The effect of high angular momenta on fissionability is preferentially studied by using complex projectiles.

\section{Mass distribution}

For highly fissionable nuclei the valley in the double peaked mass yield curve characteristic for low energy induced fission of these elements is gradually filled in when the bombarding energy increases towards $50-100 \mathrm{MeV}$. From the relatively few data available the mass yield curves above $100 \mathrm{MeV}$ bombarding energy have been assumed to be single peaked with a flat maximum extending over 30 to 40 mass numbers and reflection symmetric ${ }^{23}$ except for the extreme light and heavy masses (Fig. 2.74 in ref. ${ }^{24}$ ). But as first indicated by RUdSTAM and PAPPAS ${ }^{17}$ in an analytical treatment of $170 \mathrm{MeV}$ proton induced fission based on a model of two different modes of fission: symmetric and asymmetric, the mass distribution should be neither single peaked nor reflection symmetric. This has recently been verified experimentally by PAPPAS and HAGEBø ${ }^{19}$, and their mass yield curve is given in Fig. 2. At higher excitation energies only incomplete experimental information is available, thus similar details of the mass distribution above $200 \mathrm{MeV}$ are not available.

The discrepancy between the yields at the wings and the reflected ones increases with energy. This is mainly due to a relative increase of the yields in the light wing, while the yields in the heavy wing are relatively more independent of energy. (Fig. 2.74 in

20 S. B. Kowalski, Doctoral Thesis, University of Paris, Paris 1963.

21 V. A. Konshin, E. S. Matusevich, and V. I. Regchshevsky, Proc. Sympos. on Physics and Chemistry of Fission II, 349, IAEA, Vienna (1965).

22 G. A. Рік-P Існак, Soviet Phys.-JETP 7, 238 [1958].

23 Reflection symmetric, i. e. reflection of yields around a most probable mass.

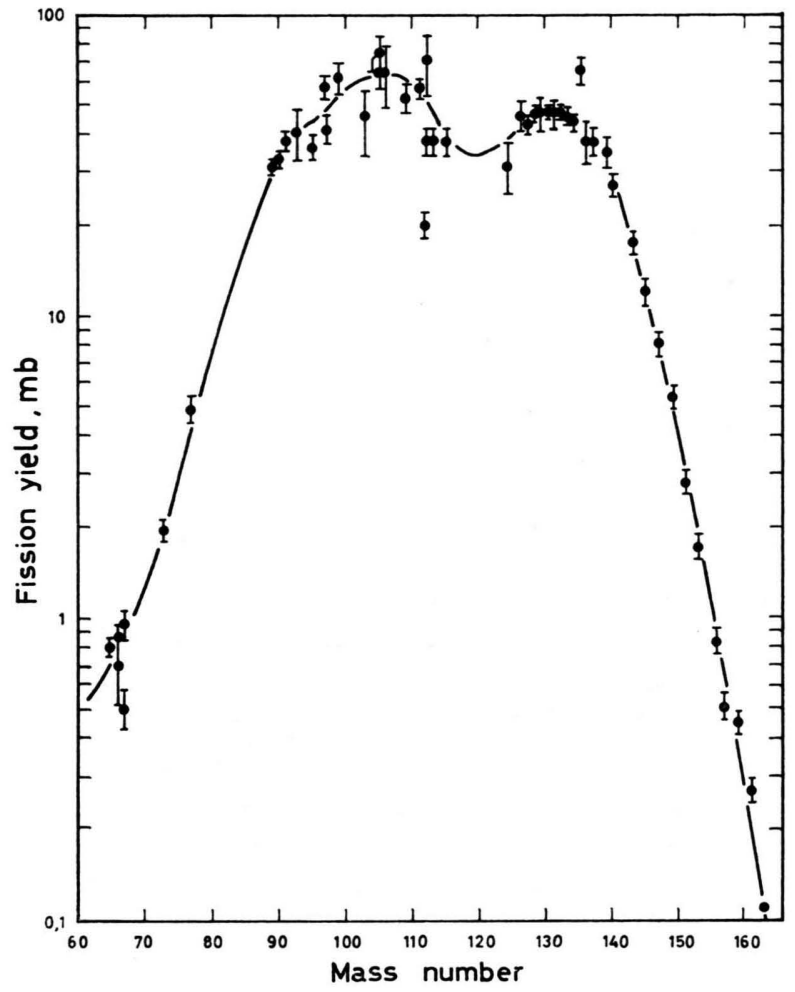

Fig. 2. Mass yield curve for fission of uranium induced by $170 \mathrm{MeV}$ protons ${ }^{19}$.

ref. ${ }^{24}$ ). By comparing crude mass yield distributions at various energies FRIEDLANDER ${ }^{25}$ shows (Fig. 3 ) that the "peak" of the distribution moves slowly towards lower mass numbers with increasing proton energy from $A$ about 118 at $100 \mathrm{MeV}$ to $\mathrm{A}$ about 107 at $28 \mathrm{GeV}$. This trend can be ascribed to a broadening of the distribution of cascade residuals and to higher average deposition energies.

That the mass yield curves in high energy induced fission show both symmetric and asymmetric trends do not necessarily involve that these curves are results of the two modes of fission: symmetric and asymmetric. The agreement between the observed distribution and that expected by the model used by Rudstam and Pappas ${ }^{17}$ may be fortuitous. An asymmetry in the curve might also be a sum of symmetric mass splitting of fissioning nuclei with large

24 E. K. Hyde, The Nuclear Properties of the Heavy Elements Vol. III, Fission Phenomena, Prentice Hall, Inc., Englewood Clifts, N. J. (1964), p. 462.

25 G. Friedlander, Proceedings of Symposium on Physics and Chemistry of Fission II, 265, IAEA, Vienna (1965).

as P. C. Stevenson, H. G. Hicks, W. E. Nervik, and D. R. Nethaway, Phys. Rev. 111, 886 [1958]. 


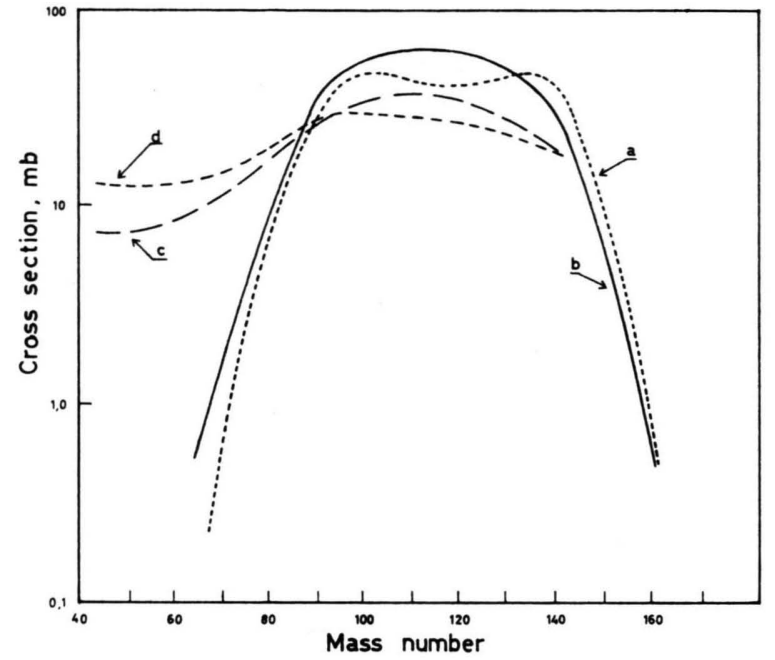

Fig. 3. Mass yield curves for uranium bombarded by protons of various energies, taken from ref. ${ }^{25}$. a) $100 \mathrm{MeV}^{26}$, b) $170 \mathrm{MeV}^{17}$, c) $2.9 \mathrm{GeV}^{25}$, d) $28 \mathrm{GeV}^{25}$.

differences in mass number and in varying aboundance and excitation energy. Thus the contribution from asymmetric splitting of nuclei can not be settled from studies of mass yield curves alone.

However, studies of charge dispersion and distribution in $170 \mathrm{MeV}$ proton induced fission of uranium point towards a real contribution from the asymmetric fission mode at least in the heavy

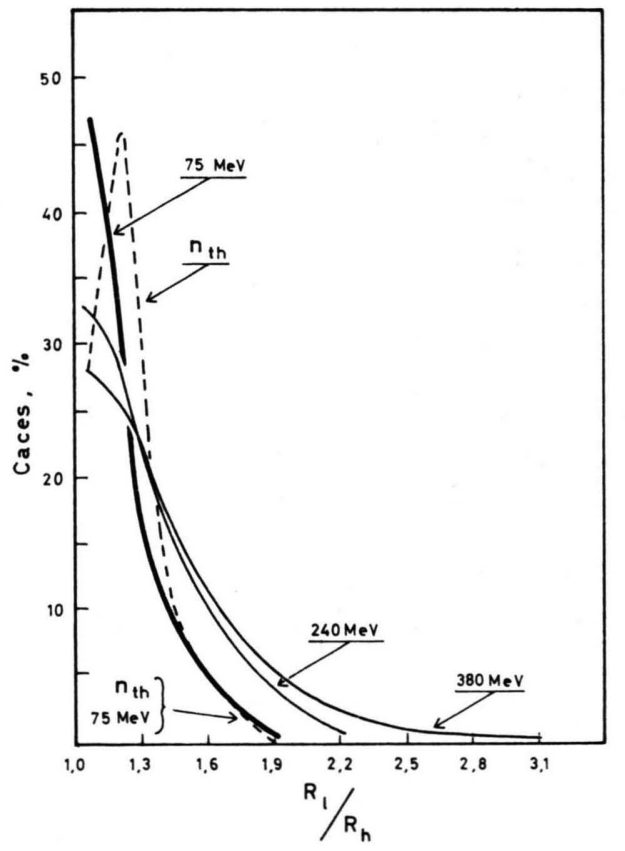

Fig. 4. Fragment range distributions from fission of uranium at different excitation energies, taken from ref. ${ }^{27}$. wing ${ }^{16}$. A support in this direction is also given by Shamov and Lozhкin ${ }^{27}$ in their studies of ranges of fission fragments in nuclear emulsion (Fig. 4). These authors show that fission of uranium is most symmetric at excitation energy $60-100 \mathrm{MeV}$ of the fissioning nucleus. However, the amount of highly asymmetric division at this energy (with range of light fragment/range of heavy fragment larger than 1.4 to 1.5) is about the same as in thermal fission. (Their observation that at higher energies the contribution from asymmetric fission increases again is hard to explain.)

The assumption, therefore, made by Rudstam and Pappas ${ }^{17}$ seems to be confirmed: Any fission nucleus at any energy can have two different modes of fission (symmetric or asymmetric) with relative probabilities depending systematically on charge and mass number and excitation energy. Those dependencies can be evaluated from experimental data from low energy fission.

As shown by Lindner and Turkevich ${ }^{7}$, when comparing their results from Monte Carlo calculations with experimental information, the fission evaporation competition $\Gamma_{\mathrm{f}} / \Gamma_{\mathrm{n}}$ in the second step of the SERBer model seems to be a function of nuclear type only and not very much on excitation energy below about $100 \mathrm{MeV}$. Thus it is most appropriate to use the results in ref. ${ }^{17}$, which are based on energy independent fission parameter

$$
\left(G=\Gamma_{\mathrm{f}} /\left(\Gamma_{\mathrm{f}}+\Gamma_{\mathrm{n}}+\Gamma_{\gamma}\right) .\right.
$$

One would then expect that the asymmetric fission mode would vanish at some excitation energy in the $100-200 \mathrm{MeV}$ region. (Fig. 2 in ref. ${ }^{17}$ ). Thus the mass yield curve will probably keep its general "structure" until the meson influence is reached.

\section{Charge Dispersion and Distribution 28}

In thermal and low energy induced fission of uranium a large amount of information is available concerning charge dispersion and distribution.

27 Quoted by N. A. Perfilov, N. S. Ivanova, O. V. Lozhrin, V. I. Ostroumov, and V. P. Shamov, Proc. Conf. Academy of Sciences of the USSR on the Peaceful Uses of Atomic Energy, Moscov (1955). Engl. trans 3, 55 [1956].

28 The nomenclature proposed by FrIEDLANDER ${ }^{25}$ will be used in this discussion, i. e.

Charge dispersion $=$ distribution of independent fission yields among the isobars at a given mass number,

Charge distribution $=$ the manner in which the nuclear charge divides itself between the two fragments in the fission act. 
The previous heavy support ${ }^{29}$ (radiochemical) to the empirical equal charge displacement rule (ECD) by Glendenin, Coryell and Edwards ${ }^{30}$ in favour of other postulates for the division of nuclear charge in fission - the charge distribution, has been enlarged by X-ray identification of charges ${ }^{31}$. In medium and high energy induced fission of bismuth the unchanged charge distribution rule (UCD), proposed by Goeckermann and Perlman ${ }^{32}$, accounts for the experimental observations. Thus it is common to assume that ECD and UCD belong to the asymmetric and the symmetric fission modes, respectively.

In medium high energy induced fission of elements thorium and heavier there have been different opinions concerning the charge division. The detailed study of fission of uranium induced by $170 \mathrm{MeV}$ protons ${ }^{16-19}$ has, however, disclosed that both the above hypotheses seem to find experimental supports.

The largest contribution from ECD is found in the heavy wing ${ }^{16}$, showing the importance of low energy modes of fission (asymmetric) where the fissioning nuclei still keep some "memory" of shell structure. The light wing, however, follows more the UCD trend, as a result of high energy deposition events ${ }^{18}$. This applies to both the neutron to proton ratios of the most probable primary charge $Z_{\mathrm{p}}$ and to the widths of the charge dispersion curves. Thus both fission modes contribute, and a mass dependent mixture of these charge distribution rules seems to be present. The mass dependence of these is therefore not quantitatively predicted by Rudstam and PAppas ${ }^{17}$. In general the experimental neutron to proton ratios, $N / Z_{\mathrm{p}}$ values, fluctuate between the limits given by the two rules. This is in qualitative agreement with what would be expected when the fissioning nuclei have a broad excitation energy spectrum.

Both charge dispersion curves observed are narrow "thermal like" gaussian curves. The width at half-maximum which is about 2.2 units in the light mass region increases slightly to 2.8 for the medium region and decreases to 2.2 in the heavy region.

29 A. C. Pappas, Proc. Intern. Conf. Peaceful Uses of Atomic Energy 7, 19, United Nations, New York (1956).

30 L. E. Glendenin, C. D. Coryell, and R. R. Edwards, In Radiochemical Studies: The Fission Products, National Nuclear Energy Series 9, Div. IV, Edited by C. D. Coryell and N. Sugarman, McGraw-Hill Book Co., Inc., New York 1951, Paper 52.
Thus the previous explanation ${ }^{16,33}$ of a broad isotopic distribution curve for iodine ${ }^{14}$ to mean a very broad charge dispersion curve in this region (width $\sim 4$ charge units) is not completely justified by the more detailed investigation ${ }^{19}$. There is, however, still a significant difference $(25-30 \%)$ in the widths of the charge dispersion curves for symmetric and asymmetric fission at $170 \mathrm{MeV}$. Thus the conclusion made by Pappas and Alstad in ${ }^{16}$, partly based on work by PATE et al. ${ }^{33}$ that the width of the charge distribution curve in symmetric fission increases with increasing bombarding energy while that for asymmetric fission is independent of energy, is still valid. As shown by Friedlander ${ }^{25}$, the yield versus $N / Z$ curves for $A=131^{34}$ are double peaked in the $\mathrm{GeV}$ region. The widths of the corresponding charge dispersion curves can be estimated to $\sim 4$ and $\sim 3$ charge units for the neutron deficient and neutron rich peak, respectively. The latter peak must be ascribed to symmetric fission and its width is as

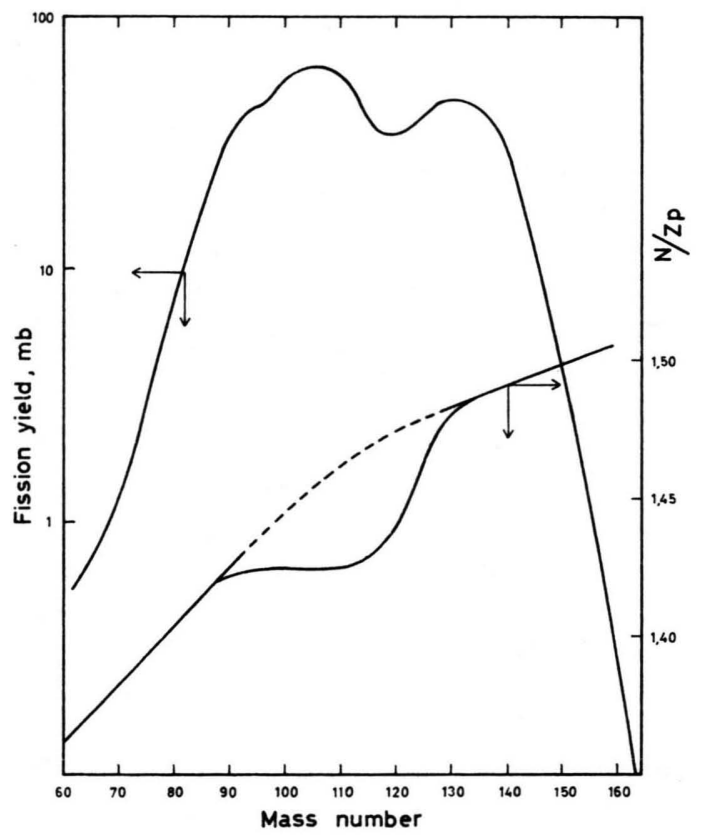

Fig. 5. The neutron to proton ratio $N / Z_{\mathrm{p}}$ for the most probable primary charge $Z_{\mathrm{p}}$ versus product mass number. Mass yield curve from Fig. 2. Both for uranium fission induced by $170 \mathrm{MeV}$ protons ${ }^{19}$.

31 L. E. Glendenin, J. P. Unik, and H. C. Grifin, Proc. Sympos. Physics and Chemistry of Fission I, 369, IAEA Vienna (1965).

32 R. H. Goecrermann and I. Perlman, Phys. Rev. 76, 628 [1949].

33 B. D. Pate, J. S. Foster, and L. Yaffe, Can. J. Chem. 36, 1691 [1958]. 
expected by extrapolation from data at lower energies. The neutron deficient peak may indicate some type of a non fission contribution ${ }^{34,35}$.

The neutron to proton ratio $N / Z_{\mathrm{p}}$ of the most probable fission products as function of mass number in $170 \mathrm{MeV}$ proton induced fission of uranium has also been studied by Pappas and Hagebø ${ }^{19}$. Their curve is reproduced in Fig. 5 on the mass yield. curve. The dip in the curve below the general trend (dotted) is in the same mass region as the symmetric peak in the mass yield curve. Thus one should conclude from position and $N / Z_{\mathrm{p}}$ ratio that symmetric fission mode is caused by high energy deposition events.

\section{Neutron emission}

Correlation studies between fission fragments and neutrons in high energy induced fission are only available for protons with $150 \mathrm{MeV}$ kinetic energy on uranium. Harding and co-workers ${ }^{36}$ report $13.1 \pm 1.8$ neutrons emitted, with only about 2.5 neutrons emitted post fission ${ }^{37}$. The majority of the neutrons are most likely emitted from nuclei which after fission give products in the middle part of the mass yield curve ${ }^{19}$. For fissioning nuclei giving products in the light wing however the radiochemical studies ${ }^{18,19}$ seem to indicate 1 . a large post fission neutron emission from light primary fission fragments, but due to low yields these neutrons are difficult to distinguish in the neutron measurements, and 2. that light products may also be formed in fission of light uranium nuclei which have evaporated many neutrons before fission.

The number of emitted neutrons is directly related to the excitation energy of the nucleus terminating the cascade. Therefore the light products are mainly the result from very high energy deposition events $\left(\sim 15 \%\right.$ compound formation is expected ${ }^{2}$ ). This conclusion is supported by a quite different approach recoil studies of fission fragments from uranium bombarded with $150 \mathrm{MeV}$ protons by Noshkin and Sugihara ${ }^{38}$ and with $450 \mathrm{MeV}$ protons by SugarMAN et al. ${ }^{39}$. Both these groups have shown that the

34 G. Friedlander, L. Friedman, B. Gordon, and L. Yaffe, Phys. Rev. 129, 1809 [1963].

35 G. Rudstam, P roc. Sympos. on Physics and Chemistry of Fission II, 323, IAEA, Vienna (1965).

36 G. N. Harding, Proc. Phys. Soc. London A 69, 330 [1956]. - G. N. Harding and F. J. M. FArlex, Proc. Phys. Soc. London A 69, 853 [1956]. - D. M. Skyrme and G. N. Harding, Nuovo Cim. 9, 1082 [1958]. excitation energies of residual nuclei leading to light fragments are about twice the energies of nuclei leading to heavy fragments.

A study of the number of prefission neutrons emitted versus mass number of the fission product is possible from the radiochemical yield measurements by PAPpas and HAGebø ${ }^{19}$. Their results are reproduced as curve A in Fig. 6 and the energy effect on the symmetric peak is also here very pronounced and supports the results from the charge distribution study (Fig. 5). One could therefore make the conclusion that the products concerned must be the result of events from the higher part of the excitation energy distribution of the nuclei terminating the cascade.

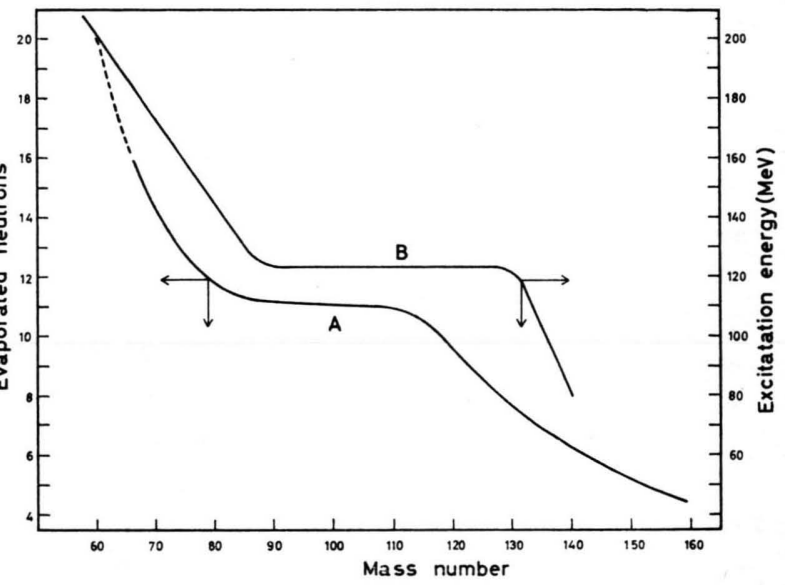

Fig. 6. Curve A: Pre fission emission of neutrons versus fission product mass number for fission of uranium with $170 \mathrm{MeV}$ protons ${ }^{19}$. Curve B: Cascade deposition energy for most probable primary charge $Z_{\mathrm{p}}$ versus fission product mass number for fission of uranium with $450 \mathrm{MeV}$ protons, taken from ref. ${ }^{39}$.

The shape of curve A in Fig. 6 is in qualitative agreement with that of curve $B$. The latter gives the variation with mass number of the cascade deposition energy for the most probable charge in $450 \mathrm{MeV}$ proton fission of uranium. This curve is determined by Sugarman et al. on the basis of recoil studies ${ }^{39}$. That the horisontal part of curve $B$ is displaced towards higher mass numbers is a result of the expected larger contribution from products from

37 Thus 2.5 neutrons are in general assumed to be emitted from the primary fission fragments in the following discussion.

38 V. E. Noshkin and T. T. Sugrhara, J. Inorg. Nucl. Chem. 27, 959 [1965].

39 N. Sugarman, H. Münzel, J. A. Panolin, K. Wielgoz, M. V. Ramaniah, G. Lange, and E. Lopez-Menchero, Phys. Rev. in press [1966]. 
higher deposition energy events. This curve gives directly the neutron emission curve for $450 \mathrm{MeV}$ fission by using the left ordinate $(\sim 10 \mathrm{MeV}$ separation and kinetic energy per neutron emitted).

The agreement obtained by two so different approaches as fission yield measurements ${ }^{19}$ and recoil studies ${ }^{39}$ is very encouraging and supports the validity of results obtained by either methods and the conclusions drawn in this paper.

\section{Conclusion}

The results of the present investigation are summarized schematically in Fig. 7, where the origin to the different parts of the mass yield curve in fission of uranium with $170 \mathrm{MeV}$ protons is illustrated. At the

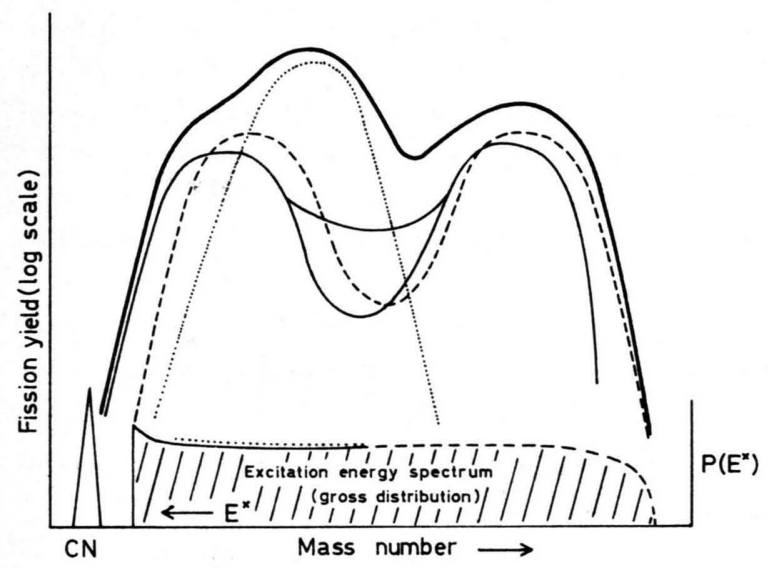

Fig. 7. Schematic representation of the origin to the different parts of mass yield curve for fission of uranium with $170 \mathrm{MeV}$ protons. (See text; B $--; \mathrm{C}-; \mathrm{D} \cdots \cdots$.)

bottom of the figure is drawn also schematically the gross distribution of deposition energy (taken from ref. ${ }^{2}$ ). The small peak to the left represents the about $15 \%$ compound nucleus formation which takes place $^{2}$.

A number of interesting features is seen from this figure:

A) The asymmetric fission peaks are in general the result of the asymmetric fission mode which occurs at all excitation energies. The charge distribution will therefore follow more close the equal charge displacement rule (ECD) than the unchanged charge distribution rule (UCD).

B) The heavy wing is due to fission of heavier nuclei, i. e. to low or moderate energy deposition events (low energy fission). The light fragment from these events will be drawned under the light peak. In the heavy wing will the ECD rule be most pronounced.

C) The light wing is due to high deposition energy events, including compound nucleus formation. The heavy products from these events will be drawned under the heavy peak. In the light wing will the UCD rule be most pronounced.

D) The symmetric peak is due to events from the higher part of the deposition energy distribution and is in general the result of the symmetric fission mode. Thus the charge distribution will follow more closely the UCD than the ECD rule.

Thus fission of heavy elements induced by protons with about $200 \mathrm{MeV}$ kinetic energy is not different in any essential way from fission induced by particles of a few tens of $\mathrm{MeV}$ kinetic energy. The maximum fissionability of uranium is reached at about $200 \mathrm{MeV}$ bombarding energy. From then on other processes (or mechanisms) than fission start contributing.

\section{Acknowledgements}

It is a pleasure for the author to use this occasion to thank his collaborators in the "Oslo-Uppsala" group, Drs. J. Alstad, E. Hagebo, A. Kjelberg, R. Rudstam and P. AagaARD, whose work has helped so much in our understanding of fission induced by protons with a few hundred MeV kinetic energy. Special thanks are due to Drs. J. Alstad and E. Hagebø for valuable discussions and comments in connection with the thoughts presented in this paper.

The experimental work of the group was performed at the Gustaf Werner Institute, Uppsala, Sweden, and on behalf of the group the author wishes to express his thanks to Professor The Svedberg, director of the institute, for his interest in these studies and for all facilities provided during these years.

The work by the Oslo-Uppsala group has had financial support from the CERN Theoretical Study Group (before CERN moved to Geneva), The Royal Norwegian Council for Scientific and Industrial Research, the Nansen Foundation in Norway, and the Swedish Council for Atomic Research (after CERN moved to Geneva). The present paper has been supported by the Nansen Foundation in Norway. All this assistance is greatly appreciated.

On the occasion of the 60th anniversary of Professor W. GentNer it is a great pleasure for the author to dedicate this paper to him commemorating his interest in high energy fission studies and also his help and devotion for the successful development of the CERN Nuclear Chemistry Group, originally started as the Oslo-Uppsala group. 